\title{
Greedy Rankings and Arank Numbers
}

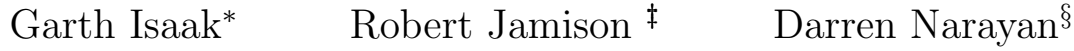

March 19, 2009

\begin{abstract}
A ranking on a graph is an assignment of positive integers to its vertices such that any path between two vertices of the same rank contains a vertex of strictly larger rank. A ranking is locally minimal if reducing the rank of any single vertex produces a non ranking. A ranking is globally minimal if reducing the ranks of any set of vertices produces a non ranking. A ranking is greedy if, for some ordering of the vertices, it is the ranking produced by assigning ranks in that order, always selecting the smallest possible rank. We will show that these three notions are equivalent. If a ranking satisfies one property it satisfies all three. As a consequence of this and known results on arank numbers of paths we improve known upper bounds for on-line ranking.
\end{abstract}

\section{Introduction}

A ranking on a graph is an assignment of positive integers to its vertices such that any path between two vertices of the same rank contains a vertex of strictly larger rank. A ranking is locally minimal if reducing the rank of any single vertex produces a non-ranking. A ranking is globally minimal if reducing the ranks of any set of vertices produces a non-ranking. A ranking is greedy if, for some ordering of the vertices, it is the ranking produced by assigning ranks in that order, always selecting the smallest possible rank. We will show that these three notions are equivalent. If a ranking satisfies one property it satisfies all three.

The rank number of a graph is the minimum size of a largest label among all rankings. Such a ranking will necessarily be minimal. Ranking was introduced in [5] motivated by separator trees and VLSI layout. The arank number of a graph is the maximum size of a largest label among all minimal rankings. The arank number was introduced in [4] using locally minimal as the definition of minimal. For on-line ranking, the vertices are presented in some order and a rank is assigned to each vertex in turn based only on the information from the previously presented vertices. For each choice of an on-line algorithm to assign the ranks, there is a worst case ordering, producing the largest label among all possible orderings. An algorithm that minimizes this worst case label is optimal and this value is called the on-line ranking number. On-line ranking was introduced in [10]. Given the equivalences above the arank number is the largest label for the worst ordering when the greedy algorithm is used. Hence the arank number is an upper bound for the online ranking number. Clearly the ranking number is a lower bound for on-line ranking number. As noted above, using known result for aranking we give slight improvements to known upper bounds for online ranking paths and cycles.

The path condition applied to edges shows that rankings are proper colorings. For ordinary graph coloring we can similarly consider locally minimal, globally minimal, and greedy colorings by replacing 'rank' with

\footnotetext{
*Department of Mathematics, Lehigh University, Bethlehem, PA 18015

${ }^{\dagger}$ Department of Mathematical Sciences, Clemson University, Clemson, SC 29634

$\ddagger$ Affiliated Professor, University of Haifa

$\S$ School of Mathematical Sciences, Rochester Institute of Technology, Rochester, NY 14623
} 
'color' and 'ranking' with 'proper coloring'. The equivalence of globally minimal and locally minimal in this case is clear, if reducing the colors on a set of vertices produces a coloring then reducing only a vertex whose original color was among the smallest also produces a proper coloring. The equivalence of minimal and greedy for coloring is also fairly straightforward. This seems to have first been shown in [3]. For rankings we need to work a little harder to show the equivalences as we need to consider all paths whose ends have the same rank that contain a vertex whose rank has been reduced.

\section{Notions of Minimality}

We first introduce some basic notation formalizing the descriptions given in the introduction.

If $G$ is a simple, undirected graph, a labeling $f: V \longrightarrow \mathbb{Z}^{+}$is a ranking if $f(u)=f(v)$ implies that every $u-v$ path contains a vertex $w$ such that $f(w)>f(u)$. A ranking $f$ is locally minimal if for all $u \in V(G)$, a labeling $g$ satisfying $g(v)=f(v)$ when $u \neq v$ and $g(u)<f(u)$, is not a ranking. A ranking $f$ is globally minimal if a labeling $g$ satisfying $g(v) \leq f(v)$ for all $v$ with at least one strict inequality is not a ranking. Using the order $f \preceq g$ if and only if $f(v) \leq g(v)$ for every $v \in V(G)$ we note that $f$ is globally minimal exactly it is minimal with respect to $\preceq$.

Given a ranking $f$ on $G$, a vertex $s$ of $G$ is a drop vertex of order $k$ for $f$ iff the function $f^{*}$ defined by

$$
f^{*}(s):=f(s)-k \text { and } f^{*}(v):=f(v) \text { for all } v \neq s \text { in } V
$$

is again a ranking. In this case, we will call $f^{*}$ a $d r o p$ of $f$. Using this notion, a ranking is locally minimal exactly when it has no drop vertex.

The equivalence of locally minimal and globally minimal rankings was first stated in [6] with the proof deferred to an unpublished manuscript [7]. We include the proof here for completeness.

Theorem 1 Let $f$ be a ranking on a graph $G=(V, E)$. Then $f$ is locally minimal if and only if it is globally minimal.

Proof. That every globally minimal ranking is locally minimal is immediate from the definitions. To show the converse we will show if $f$ is not globally minimal then it is not locally minimal by showing that if $g$ is a ranking with $g \preceq f$ and $g \neq f$, then there is a drop $f^{*}$ of $f$ with $g \preceq f^{*} \preceq f$.

Consider the set of differing vertices $D:=\{v \in V: g(v)<f(v)\}$. Let $m=\min \{f(v): v \in D\}$. Let $s$ be a vertex in $D$ such that $f(s)=m$. We shall show that $s$ is a drop vertex of order $k:=f(s)-g(s)$. Indeed, define the function $f^{*}$ by

$$
f^{*}(s):=g(s) \text { and } f^{*}(v):=f(v) \text { for all } v \neq s \text { in } V
$$

We must show that $f^{*}$ is again a ranking. Let $u \neq v$ be vertices of $G$ with $f^{*}(u)=f^{*}(v)$, and let $P$ be a path between $u$ and $v$. We must show that $P$ contains a vertex $w$ with $f^{*}(w)>f^{*}(u)$. First suppose that neither $u$ or $v$ is $s$, and note that $f(u)=f^{*}(u)=f(v)=f^{*}(v)$. Since $f$ is a ranking, there is a $w$ on $P$ with $f(w)>f(u)=f(v)$. If $w \neq s$, we are done since $f^{*}(w)=f(w)>f(u)=f^{*}(u)$. Thus suppose $w=s$. Then $f(s)>f(u)=f(v)$, so by the minimal choice of $m$ and $s$, it follows that $f(u)=g(u)=g(v)=f(v)$. Since $g$ is a ranking, there is a vertex $x$ on $P$ with $g(x)>g(u)=g(v)$. If $x=s$, then $f^{*}(x)=g(x)>g(u)=f(u)=f(v)$ as desired. If $x \neq s$, then $f^{*}(x)=f(x) \geq g(x)>g(u)=f(u)=f(v)$ as desired.

Hence we may assume without loss of generality that $u=s$. Then $f(v)=f^{*}(v)=f^{*}(s)=g(s)<f(s)$. By the minimal choice of $m$ and $s$, it follows that $f(v)=g(v)$. Since $g$ is a ranking and $g(s)=g(v)$, there must be a vertex $w$ on $P$ with $g(w)>g(s)$. Thus since $w \neq s$, we have

$$
f^{*}(u)=g(s)<g(w) \leq f(w)=f^{*}(w)
$$


as desired. Therefore $f^{*}$ is a ranking and hence a drop of $f$ with $g \leq f^{*}<f$.

We can now refer to without specifying locally or globally. However we will continue to do so in order to make clear what we are assuming in the proofs.

\section{$3 \quad$ Minimal $=$ Greedy}

We now prove that minimal rankings are greedy. It is convenient to use globally minimal rather than locally minimal. The proof that greedy rankings are globally minimal can easily be used to also show that they are locally minimal. However, for the converse it seems that we need to use globally minimal rather than locally minimal or else the proof also needs to essentially include the proof of Theorem 1.

We will use the fact that a ranking restricted to an induced subgraph is a ranking of the induced subgraph. We will also use a special case of a basic lemma from [4]: two vertices with the largest rank must be in different components as any path connecting them would not contain a vertex of larger rank.

Theorem 2 Let $f$ be a ranking on a graph $G=(V, E)$. Then $f$ is greedy if and only if it is globally minimal.

Proof. Only if: Index the vertices so the greedy algorithm applied to the ordering $v_{1}, v_{2}, \ldots, v_{n}$ produces the ranking $f$. If $f$ is not globally minimal consider $g \prec f$ and let $i$ be the smallest index with $g\left(v_{i}\right)<f\left(v_{i}\right)$. Since $g$ is a ranking of the subgraph induced by $\left\{v_{1}, v_{2}, \ldots, v_{i}\right\}$ and $g\left(v_{j}\right)=f\left(v_{j}\right)$ for $j=1,2, \ldots, i-1$ the greedy algorithm could have assigned $v_{i}$ the rank $g\left(v_{i}\right)$, contradicting the assumption that the the greedy algorithm yields the ranking $f$ on this ordering.

If: Consider $f$ which is globally minimal. Select any indexing of the vertices so that $f\left(v_{i}\right) \leq f\left(v_{j}\right)$ whenever $i \leq j$ and apply the greedy algorithm to the ordering $v_{1}, v_{2}, \ldots, v_{n}$. Let $g$ be the resulting ranking. We will show that $g \preceq f$. Then since $f$ is globally minimal we will have $g=f$, showing that $f$ is greedy.

If $g \nprec f$, let $i$ be the smallest index such that $g\left(v_{i}\right)>f\left(v_{i}\right)$. Since the greedy algorithm could not assign the rank $f\left(v_{i}\right)$ to $v_{i}$ there must be a vertex $v_{j}$ with $j<i$ and $g\left(v_{j}\right)=f\left(v_{i}\right)$ such that there is a $v_{j}-v_{i}$ path in the subgraph induced by $\left\{v_{1}, v_{2}, \ldots, v_{i}\right\}$ containing no vertices given rank greater than $f\left(v_{i}\right)$ by $g$. By the choice of $i$ we have $f\left(v_{k}\right)=g\left(v_{k}\right)$ for $k=1,2, \ldots, i-1$. So $f\left(v_{i}\right)=f\left(v_{j}\right)$ and there is a path in the subgraph induced by $\left\{v_{1}, v_{2}, \ldots, v_{i}\right\}$ containing no vertices given rank greater than $f\left(v_{i}\right)$ by $f$. Since $v_{i}$ and $v_{j}$ are in the same component of the subgraph induced by $\left\{v_{1}, v_{2}, \ldots, v_{i}\right\}$, they cannot have largest rank. So $f\left(v_{k}\right)>f\left(v_{i}\right)=f\left(v_{j}\right)$ for some $k \in\{1,2, \ldots, i\}$. But this contradicts the selection of the indexing.

\section{Upper bounds for the on-line ranking number}

We use the following notation: $\chi_{r}(G)$ for the ranking number of $G, \psi_{r}(G)$ for the arank number of $G$ and $\chi_{r}^{*}(G)$ for the on-line ranking number of $G$.

As noted in the introduction, our results show that the arank number provides an upper bound on the online ranking number and the ranking number is a trivial lower bound. With our notation this becomes

Corollary 3 For any graph $G, \chi_{r}(G) \leq \chi_{r}^{*}(G) \leq \psi_{r}(G)$.

A problem of interest is to determine the on-line ranking number of paths $P_{n}$ and cycles $C_{n}$ on $n$ vertices. The arank number of paths was determined as $\psi_{r}\left(P_{n}\right)=\left\lfloor\log _{2}(n+1)\right\rfloor+\left\lfloor\log _{2}\left(n+1-\left(2^{\left.\log _{2} n\right\rfloor-1}\right)\right)\right\rfloor$ in [9]. For cycles, it was shown in [8] that $\psi_{r}\left(C_{n}\right)$ either equals $\psi_{r}\left(P_{n}\right)$ or $\psi_{r}\left(P_{n}\right)+1$.

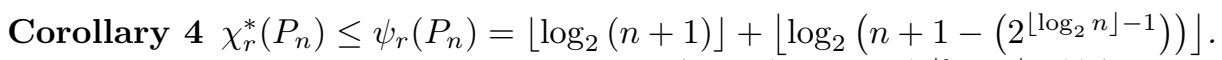

$\psi_{r}\left(C_{n}\right) \leq \psi_{r}\left(P_{n}\right)+1=\left\lfloor\log _{2}(n+1)\right\rfloor+\left\lfloor\log _{2}\left(n+1-\left(2^{\left\lfloor\log _{2} n\right\rfloor-1}\right)\right)\right\rfloor+1$. 
Corollary 4 improves on the bounds $\chi_{r}^{*}\left(P_{n}\right) \leq 2\left\lfloor\log _{2} n\right\rfloor+1$ and $\chi_{r}^{*}\left(C_{n}\right) \leq 2\left\lfloor\log _{2} n\right\rfloor+1$ from [1] when $n \geq 16$

The best lower bounds for online ranking paths and cycles are $1.619 \log _{2} n-1 \leq \chi_{r}^{*}\left(P_{n}\right)$ [2] and $\left\lfloor\log _{2}(n+1)\right\rfloor \leq \chi_{r}^{*}\left(C_{n}\right)$ when $n=2^{k}$ and $\left\lfloor\log _{2}(n+1)\right\rfloor+1 \leq \chi_{r}^{*}\left(C_{n}\right)$ when $n \neq 2^{k}[1]$

\section{References}

[1] E. Bruoth and M. Horňák, On-line ranking number for cycles and paths, Discussiones Mathematicae, Graph Theory, vol 19. pp. 175-197, 1999.

[2] E. Bruoth and M. Horňák, A lower bound for on-line ranking number of a path, Discrete Math. 307 (2007), 1347-1355.

[3] P. Erdos, W.R. Hare, S.T. Hedetniemi, R. Laskar, On the equality of the Grundy and ochromatic numbers of a graph, J. Graph Theory 11 (1987), 157 - 159.

[4] J. Ghoshal, R. Laskar, and D. Pillone. Minimal rankings, Networks, Vol. 28, (1996), 45-53.

[5] A. Iyer, H.D. Ratliff, and G. Vijayan, Optimal node ranking of trees, Inform. Process. Lett. 28(1988), $225-229$.

[6] R.E. Jamison, Coloring parameters associated with rankings of graphs, Cong. Num. 164 (2003), 111 127.

[7] R.E. Jamison, On the ranking poset of a graph, unpublished manuscript.

[8] V. Kostyuk and D.A. Narayan, Maximum minimal rankings of cycles, to appear in Ars Combinatoria.

[9] V. Kostyuk, D.A. Narayan, and V.A. Williams, Minimal rankings and the arank number of a path, Discrete Math 306 (2006) 1991-1996.

[10] Zs. Tuza and M. Voigt, Ranking problems on graphs, Manuscript, 1995. 